CRYSTALLOGRAPHIC COMMUNICATIONS

Received 20 April 2015

Accepted 18 May 2015

Edited by M. Weil, Vienna University of Technology, Austria

Keywords: crystal structure; hydrous ternary sulfates; hydrothermal synthesis; hydrogen bonding

CCDC reference: 1401662

Supporting information: this article has

supporting information at journals.iucr.org/e

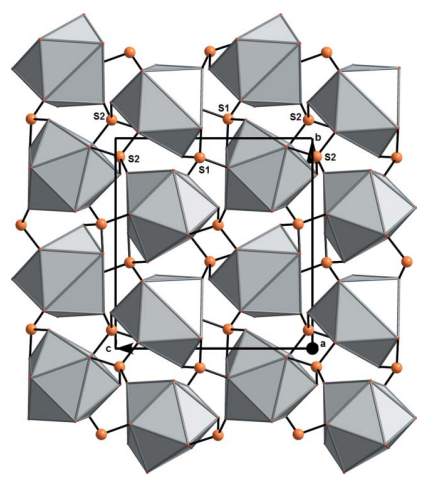

OPEN $\odot$ ACCESS

\section{Crystal structure of $\mathrm{NH}_{4}\left[\mathrm{La}\left(\mathrm{SO}_{4}\right)_{2}\left(\mathrm{H}_{2} \mathrm{O}\right)\right]$}

\author{
Meriem Benslimane, ${ }^{\mathrm{a} *}$ Yasmine Kheira Redjel, ${ }^{\mathrm{a}}$ Hocine Merazig ${ }^{\mathrm{a}}$ and Jean-Claude \\ Daran $^{\text {b }}$
}

a Unité de Recherche de Chimie de l'Environnement et Moléculaire Structurale, Faculté des Sciences Exactes, Département de Chimie, Université de Constantine 1, 25000 Constantine, Algeria, and ${ }^{\mathbf{b}}$ Laboratoire de Chimie de Coordination, UPR-CNRS 8241, 05 route de Narbonne, 31077 Toulouse Cedex 4. *Correspondence e-mail: b_meriem80@yahoo.fr

The principal building units in the crystal structure of ammonium aquabis(sulfato)lanthanate(III) are slightly distorted $\mathrm{SO}_{4}$ tetrahedra, $\mathrm{LaO}_{9}$ polyhedra in the form of distorted tricapped trigonal prisms, and $\mathrm{NH}_{4}{ }^{+}$ions. The $\mathrm{La}^{3+}$ cation is coordinated by eight $\mathrm{O}$ atoms from six different sulfate tetrahedra, two of which are bidentate coordinating and four monodentate, as well as one $\mathrm{O}$ atom from a water molecule; each sulfate anion bridges three $\mathrm{La}^{3+}$ cations. These bridging modes result in the formation of a three-dimensional anionic $\left[\mathrm{La}\left(\mathrm{SO}_{4}\right)_{2}\left(\mathrm{H}_{2} \mathrm{O}\right)\right]^{-}$framework that is stabilized by $\mathrm{O}-\mathrm{H} \cdots \mathrm{O}$ hydrogenbonding interactions. The disordered ammonium cations are situated in the cavities of this framework and are hydrogen-bonded to six surrounding $\mathrm{O}$ atoms.

\section{Chemical context}

Three-dimensional framework materials are characterized by their structural diversity. They are of continuing interest as a result of their technologically important properties and potential applications in catalysis, ion-exchange, adsorption, intercalation, and radioactive waste remediation (Szostak, 1989; Cheetham et al., 1999; Rosi et al., 2003; Ok et al., 2007). Many materials showing such functional features contain structurally versatile cations, in particular heavier metal cations with large coordination spheres. Among many other cations, lanthanide cations have been used widely, since they exhibit high coordination numbers and can show a large topological diversity in the resulting framework structures (Bataille \& Louër, 2002; Wickleder, 2002; Yuan et al., 2005). One of the most promising synthetic methods for the preparation of compounds with framework structures is the hydrothermal (or solvothermal) reaction technique (Feng et al., 1997; Natarajan et al., 2000) in which mineralizers such as acids or bases are introduced to increase the solubility and reactivity of the reagents (Laudise, 1959; Laudise \& Ballman, 1958). Moreover, organic or inorganic templates are used to direct the topologies of the framework structures and the concomitant physical and chemical properties of the products (Szostak, 1989; Breck, 1974; Barrer, 1982). Thus, we have tried to utilize the hydrothermal technique to react a lanthanide cation $\left(\mathrm{La}^{3+}\right)$ with sulfuric acid in the presence of $\mathrm{NH}_{4} \mathrm{OH}$ and 3-aminobenzoic acid as a template to prepare higher dimensional framework materials. However, in the present case the organic template was not incorporated in the resultant crystal structure of the title compound, $\mathrm{NH}_{4}\left[\mathrm{La}\left(\mathrm{SO}_{4}\right)_{2}\left(\mathrm{H}_{2} \mathrm{O}\right)\right]$, which represents a new hydrate form. Other members in the system $\mathrm{NH}_{4}{ }^{+} / \mathrm{La}^{3+} / \mathrm{SO}_{4}{ }^{2-} /\left(\mathrm{H}_{2} \mathrm{O}\right)$ are two forms of anhydrous $\left(\mathrm{NH}_{4}\right)\left[\mathrm{La}\left(\mathrm{SO}_{4}\right)_{2}\right]$ (Sarukhanyan et al., 1984a; Bénard- 


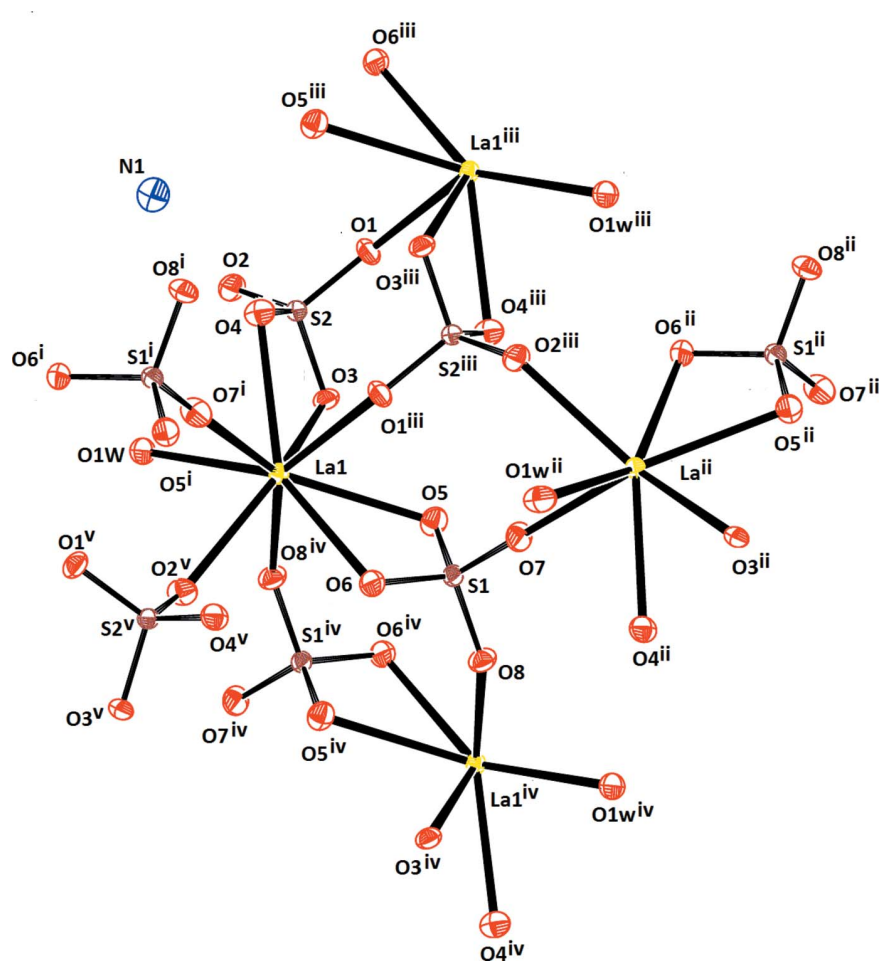

Figure 1

The principal building units, $\mathrm{LaO}_{9}$ polyhedra and $\mathrm{SO}_{4}$ tetrahedra, in the crystal structure of $\left(\mathrm{NH}_{4}\right)\left[\mathrm{La}\left(\mathrm{SO}_{4}\right)_{2}\left(\mathrm{H}_{2} \mathrm{O}\right)\right]$, showing the atom-labelling scheme. Displacement ellipsoids are drawn at the $50 \%$ probability level. [Symmetry codes: (i) $1-x,-\frac{1}{2}+y, \frac{1}{2}-z$; (ii) $1-x, \frac{1}{2}+y, \frac{1}{2}-z$; (iii) $1-x$, $2-y,-z$; (iv) $2-x, 2-y, 1-z$; (v) $x, \frac{3}{2}-y, \frac{1}{2}+z$.]

Rocherullé et al., 2001), $\left(\mathrm{NH}_{4}\right)_{5}\left[\mathrm{La}\left(\mathrm{SO}_{4}\right)_{4}\right]$ (Niinisto et al., $1980)$ and $\left(\mathrm{NH}_{4}\right)\left[\mathrm{La}\left(\mathrm{SO}_{4}\right)_{2}\left(\mathrm{H}_{2} \mathrm{O}\right)_{4}\right]$ (Keppert et al., 1999).

Sulfates with an $A^{+}: \operatorname{Ln}^{3+}\left(A^{+}=\right.$alkaline ions, $L n^{3+}=$ lanthanide ions) ratio of 1:1 are one of the best investigated groups among hydrous ternary sulfates. They crystallize either as monohydrates (Blackburn \& Gerkin, 1995; Barnes, 1995; Iskhakova et al., 1985a) or tetrahydrates (Eriksson et al., 1974), and in few cases also as dihydrates (Kaučič et al., 1985; Iskhakova \& Trunov, 1985). The tetrahydrates are mainly found for the bigger monovalent ions $\mathrm{Cs}^{+}, \mathrm{NH}_{4}{ }^{+}$, and $\mathrm{Rb}^{+}$. For the smaller $A^{+}$ions such as $\mathrm{Na}^{+}$, the monohydrate becomes dominant.

\section{Structural commentary}

The structure of the title compound comprises $\mathrm{LaO}_{9}$ polyhedra and $\mathrm{SO}_{4}$ tetrahedra as the principal building units (Fig. 1), forming an anionic $\left[\mathrm{La}\left(\mathrm{SO}_{4}\right)_{2}\left(\mathrm{H}_{2} \mathrm{O}\right)\right]^{-}$framework by sharing common edges and vertices (Fig. 2). The $\mathrm{NH}_{4}^{+}$ counter-cations are situated in the cavities of this framework.

The $\mathrm{La}^{3+}$ cation is coordinated by eight $\mathrm{O}$ atoms from six different sulfate tetrahedra. Two tetrahedra are in a bidentate coordination mode and four tetrahedra are in a monodentate mode. The distorted tricapped trigonal-prismatic coordination sphere is completed by one $\mathrm{O}$ atom from a water molecule. The $\mathrm{La}-\mathrm{O}$ bond lengths, ranging from 2.472 (3) to 2.637 (3) $\AA$ with 2.496 (3) $\AA$ to the water molecule, and the

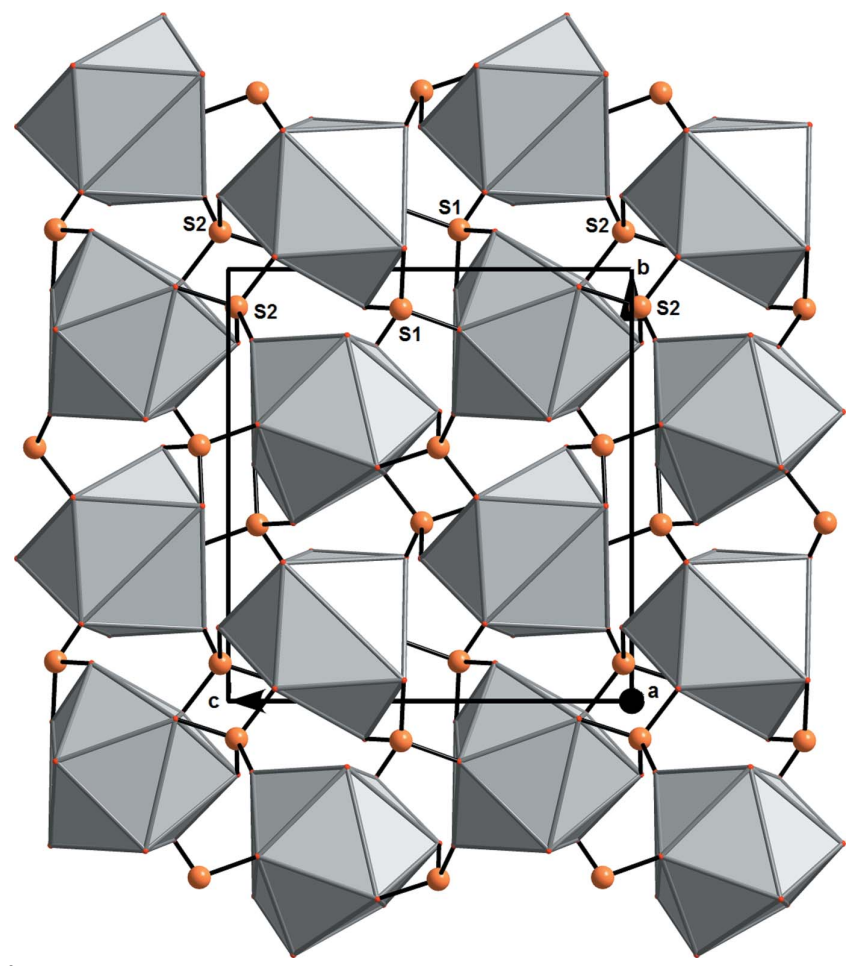

Figure 2

The connection of $\mathrm{LaO}_{9}$ polyhedra and $\mathrm{SO}_{4}$ tetrahedra in the crystal structure of $\left(\mathrm{NH}_{4}\right)\left[\mathrm{La}\left(\mathrm{SO}_{4}\right)_{2}\left(\mathrm{H}_{2} \mathrm{O}\right)\right]$, viewed along the $a$ axis.

$\mathrm{O}-\mathrm{La}-\mathrm{O}$ angles, ranging from $53.55(8)$ to $145.43(9)^{\circ}$, are similar to the analogous distances found in $\mathrm{NaLa}\left(\mathrm{SO}_{4}\right)_{2} \cdot \mathrm{H}_{2} \mathrm{O}$ (Blackburn \& Gerkin, 1995). The ninefold coordination of $\mathrm{La}^{3+}$ in $\mathrm{NH}_{4}\left[\mathrm{La}\left(\mathrm{SO}_{4}\right)_{2}\left(\mathrm{H}_{2} \mathrm{O}\right)\right]$ is typical for the majority of monohydrated alkali rare earth sulfate complexes and of rare earth complexes in general. For early members of the rare earth sulfate series, the coordination number of nine is realized, e.g. for $\mathrm{Ce}, \mathrm{Pr}, \mathrm{La}$ and $\mathrm{Nd}$ (Blackburn \& Gerkin, 1994, 1995; Iskhakova et al., 1985b, 1988). For later members of the sulfate series, such as Gd (Sarukhanyan et al., 1984b), the coordination number decreases to eight, presumably in association with the lanthanide contraction. There are two sulfur atoms (S1, S2) in the asymmetric unit of the title compound, both with very similar $\mathrm{S}-\mathrm{O}$ bond lengths in the ranges 1.465 (3)-1.488 (3) and 1.468 (3)-1.490 (3) §, respectively. The range of $\mathrm{O}-\mathrm{S}-\mathrm{O}$ bond angles, 106.04 (16)$110.89(19)^{\circ}$ for $\mathrm{S} 1$ and $104.70(16)-111.52(17)^{\circ}$ for $\mathrm{S} 2$, reflect the distortion of the two sulfate tetrahedra. Each $\mathrm{SO}_{4}$ anion bridges three $\mathrm{La}^{3+}$ cations (Fig. 2).

\section{Supramolecular features}

The bridging modes of the $\mathrm{O}$ atoms result in the formation of a three-dimensional anionic framework, stabilized by $\mathrm{O}-$ $\mathrm{H}$... O hydrogen-bonding interactions between the aqua ligand and the two $\mathrm{SO}_{4}$ tetrahedra (Table 1) whereby each sulfate tetrahedron establishes one hydrogen bond with the water molecule via the oxygen atom (O6 and O3) corresponding to the longest $\mathrm{S}-\mathrm{O}$ bonds. The $\mathrm{N}$ atoms are situated in the cavities of this framework. Although the $\mathrm{H}$ atoms of the 
Table 1

Hydrogen-bond geometry $\left(\AA,^{\circ}\right)$.

\begin{tabular}{|c|c|c|c|c|}
\hline$D-\mathrm{H} \cdots A$ & $D-\mathrm{H}$ & $\mathrm{H} \cdots A$ & $D \cdots A$ & $D-\mathrm{H} \cdots A$ \\
\hline $\mathrm{O} 1 W-\mathrm{H} 11 \cdots \mathrm{O} 3^{\mathrm{i}}$ & $0.84(5)$ & $1.94(5)$ & $2.717(5)$ & $153(5)$ \\
\hline $\mathrm{O} 1 W-\mathrm{H} 21 \cdots \mathrm{O} 6^{\mathrm{ii}}$ & $0.85(3)$ & $1.95(3)$ & $2.778(4)$ & $168(5)$ \\
\hline $\mathrm{N} 1 \cdots \mathrm{O} 1^{\mathrm{iii}}$ & & & $2.942(5)$ & \\
\hline $\mathrm{N} 1 \cdots \mathrm{O}^{\mathrm{ii}}$ & & & $3.036(5)$ & \\
\hline $\mathrm{N} 1 \cdots \mathrm{O}^{\text {iv }}$ & & & $2.914(5)$ & \\
\hline $\mathrm{N} 1 \cdots \mathrm{O}^{\mathrm{v}}$ & & & $2.943(5)$ & \\
\hline $\mathrm{N} 1 \cdots \mathrm{O}^{\mathrm{vi}}$ & & & $2.865(5)$ & \\
\hline $\mathrm{N} 1 \ldots \mathrm{O} 4$ & & & $2.866(5)$ & \\
\hline
\end{tabular}

Symmetry codes: (i) $-x+2, y-\frac{1}{2},-z+\frac{1}{2}$; (ii) $\quad x,-y+\frac{3}{2}, z-\frac{1}{2}$; $-x+1, y-\frac{1}{2},-z-\frac{1}{2} ; \quad$ (iv) $\quad x-1,-y+\frac{3}{2}, z-\frac{1}{2} ;$ (v) $-x+1, y-\frac{1}{2},-z+\frac{1}{2} ; \quad$ (vi) $-x+1,-y+2,-z$.

ammonium cation could not be located, the $\mathrm{N}$... O distances between 2.865 (5) and 3.036 (5) ^ strongly suggest $\mathrm{N}-\mathrm{H} \cdots \mathrm{O}$ hydrogen bonds of medium strength (Table 1). It appears most likely that the number of $\mathrm{O}$ atoms (six) in the vicinity of the $\mathrm{N}$ atom is the reason for the disorder of the ammonium cation.

\section{Synthesis and crystallization}

The title compound was obtained during the attempted preparation of a complex resulting from the hydrothermal reaction of $\mathrm{La}_{2} \mathrm{O}_{3}(0.1 \mathrm{~g}, 1 \mathrm{mmol})$ with $37 \%$ wt sulfuric acid and 3 -aminobenzoic acid $(0.048 \mathrm{~g}, 1 \mathrm{mmol})$ in the presence of $\mathrm{NH}_{4} \mathrm{OH}$ in $10 \mathrm{ml}$ water. The mixture was kept in a $23 \mathrm{ml}$ Teflon-lined steel autoclave at $433 \mathrm{~K}$ for $3 \mathrm{~d}$. After this treatment, the autoclave was cooled slowly to room temperature. Slow evaporation of the solvent at room temperature led to the formation of prismatic colourless crystals of the title compound.

\section{Refinement}

Crystal data, data collection and structure refinement details are summarized in Table 2. The oxygen-bound hydrogen atoms were located in a difference Fourier map and were refined with restraints of the $\mathrm{O}-\mathrm{H}$ bond length [0.85 (1) $\AA$ ] and $\mathrm{H} \cdots \mathrm{H}$ distances $(1.39 \AA)$ and with $U_{\text {iso }}(\mathrm{H})=1.5 U_{\text {eq }}(\mathrm{O})$. The ammonium hydrogen atoms could not be located reliably by difference Fourier methods. Several disorder models considering the hydrogen-bonding environment (see Table 1) failed, eventually leading to the exclusion of the ammonium hydrogen atoms from the refinement. The maximum and minimum peaks in the final difference Fourier map are 0.93 and $0.72 \AA$, respectively, from atom La1.

Diffraction data were collected some time ago, and merged in the corresponding crystal class. Unfortunately, the original measurement data got lost; experiments to repeat the crystal growth were unsuccessful. Therefore the crystal structure was finally solved and refined with the merged data set.

\section{Acknowledgements}

Technical support (X-ray measurements) from Université Henri Poincaré, Nancy 1, is gratefully acknowledged.
Table 2

Experimental details.

\begin{tabular}{|c|c|}
\hline \multicolumn{2}{|l|}{ Crystal data } \\
\hline Chemical formula & $\mathrm{NH}_{4}\left[\mathrm{La}\left(\mathrm{SO}_{4}\right)_{2}\left(\mathrm{H}_{2} \mathrm{O}\right)\right]$ \\
\hline$M_{\mathrm{r}}$ & 367.07 \\
\hline Crystal system, space group & Monoclinic, $P 2_{1} / c$ \\
\hline Temperature $(\mathrm{K})$ & 100 \\
\hline$a, b, c(\AA)$ & $8.4919(16), 9.978(2), 11.9268$ (19) \\
\hline$\beta\left(^{\circ}\right)$ & $128.511(10)$ \\
\hline$V\left(\mathrm{~A}^{3}\right)$ & $790.7(3)$ \\
\hline$Z$ & 4 \\
\hline Radiation type & Мо $K \alpha$ \\
\hline$\mu\left(\mathrm{mm}^{-1}\right)$ & 5.96 \\
\hline Crystal size $(\mathrm{mm})$ & $0.30 \times 0.20 \times 0.10$ \\
\hline \multicolumn{2}{|l|}{ Data collection } \\
\hline Diffractometer & Nonius KappaCCD \\
\hline Absorption correction & For a sphere (Dwiggins, 1975) \\
\hline$T_{\min }, T_{\max }$ & $0.419,0.431$ \\
\hline $\begin{array}{l}\text { No. of measured, independent and } \\
\text { observed }[I>2 \sigma(I)] \text { reflections }\end{array}$ & $2414,2414,2362$ \\
\hline$(\sin \theta / \lambda)_{\max }\left(\AA^{-1}\right)$ & 0.715 \\
\hline \multicolumn{2}{|l|}{ Refinement } \\
\hline$R\left[F^{2}>2 \sigma\left(F^{2}\right)\right], w R\left(F^{2}\right), S$ & $0.027,0.081,1.26$ \\
\hline No. of reflections & 2414 \\
\hline No. of parameters & 124 \\
\hline No. of restraints & 3 \\
\hline $\mathrm{H}$-atom treatment & $\begin{array}{l}\mathrm{H} \text { atoms treated by a mixture of } \\
\text { independent and constrained } \\
\text { refinement }\end{array}$ \\
\hline$\Delta \rho_{\max }, \Delta \rho_{\min }\left(\mathrm{e} \AA^{-3}\right)$ & $1.81,-1.48$ \\
\hline
\end{tabular}

Computer programs: COLLECT (Nonius, 1998), DENZO and SCALEPACK (Otwinowski \& Minor, 1997), SIR92 (Altomare et al., 1993), SHELXL97 (Sheldrick, 2008), ORTEP-3 for Windows and WinGX (Farrugia, 2012) and DIAMOND (Brandenburg \& Berndt, 1999).

\section{References}

Altomare, A., Cascarano, G., Giacovazzo, C. \& Guagliardi, A. (1993). J. Appl. Cryst. 26, 343-350.

Barnes, J. C. (1995). Acta Cryst. C51, 2466-2469.

Barrer, R. M. (1982). In Hydrothermal Chemistry of Zeolites. London: Academic Press.

Bataille, T. \& Louër, D. (2002). J. Mater. Chem. 12, 3487-3493.

Bénard-Rocherullé, P., Tronel, H. \& Louër, D. (2001). Mater. Sci. Forum, 378-381, 476-481.

Blackburn, A. C. \& Gerkin, R. E. (1994). Acta Cryst. C50, 835-838.

Blackburn, A. C. \& Gerkin, R. E. (1995). Acta Cryst. C51, 22152218.

Brandenburg, K. \& Berndt, M. (1999). DIAMOND. Crystal Impact GbR, Bonn, Germany.

Breck, D. W. (1974). In Zeolite Molecule Sieves: Structure, Chemistry and Use. London: Wiley and Sons.

Cheetham, A. K., Férey, G. \& Loiseau, T. (1999). Angew. Chem. Int. Ed. 38, 3268-3292.

Dwiggins, C. W. (1975). Acta Cryst. A31, 146-148.

Eriksson, B., Larsson, L. O., Niinisto, L. \& Skoglund, U. (1974). Inorg. Chem. 13, 290-295.

Farrugia, L. J. (2012). J. Appl. Cryst. 45, 849-854.

Feng, P., Bu, X. \& Stucky, G. D. (1997). Nature, 388, 735-741.

Iskhakova, L. D., Gasanov, Y. \& Trunov, V. K. (1988). Zh. Strukt. Khim. 29, 95-99.

Iskhakova, L. D., Sarukhanyan, N. L. \& Shchegoleva, T. M. (1985a). Kristallographiya, 30, 474-479.

Iskhakova, L. D., Sarukhanyan, N. L. \& Trunov, V. K. (1985b). Zh. Neorg. Khim. 30, 978-981.

Iskhakova, L. D. \& Trunov, V. K. (1985). Kristallographiya, 30, 279 283. 
Kaučič, V., Bukovec, N. \& Golič, Lj. (1985). Acta Cryst. C41, 636-638. Kepert, C. J., Junk, P. C., Skelton, B. W. \& White, A. H. (1999). Aust. J. Chem. 52, 601-615.

Laudise, R. A. (1959). J. Am. Chem. Soc. 81, 562-566.

Laudise, R. A. \& Ballman, A. A. (1958). J. Am. Chem. Soc. 80, 26552657.

Natarajan, S., Neeraj, S., Choudhury, A. \& Rao, C. N. R. (2000). Inorg. Chem. 39, 1426-1433.

Niinisto, L., Toivonen, J. \& Valkonen, J. (1980). Finn. Chem. Lett. 3, 87-92.

Nonius (1998). COLLECT. Nonius BV, Delft, The Netherlands.

Ok, K. M., Doran, M. B. \& O'Hare, D. (2007). Dalton Trans. pp. 3325-3329.
Otwinowski, Z. \& Minor, W. (1997). Methods in Enzymology, Vol. 276, Macromolecular Crystallography, Part A, edited by C. W. Carter Jr \& R. M. Sweet, pp. 307-326. New York: Academic Press. Rosi, N. L., Eckert, J., Eddaoudi, M., Vodak, D. T., Kim, J., O'Keeffe, M. \& Yaghi, O. M. (2003). Science, 300, 1127-1129.

Sarukhanyan, N. L., Iskhakova, L. D. \& Trunov, V. K. (1984a). Kristallografiya, 29, 435-439

Sarukhanyan, N. L., Iskhakova, L. D., Trunov, V. K. \& Ilyukhin, V. V. (1984b). Koord. Khim. 10, 981-987.

Sheldrick, G. M. (2008). Acta Cryst. A64, 112-122.

Szostak, R. (1989). In Molecular Sieves: Principles of Synthesis and Identification. New York: Reinhold.

Wickleder, M. S. (2002). Chem. Rev. 102, 2011-2088. 


\section{supporting information}

Acta Cryst. (2015). E71, 663-666 [doi:10.1107/S2056989015009457]

\section{Crystal structure of $\mathrm{NH}_{4}\left[\mathrm{La}\left(\mathrm{SO}_{4}\right)_{2}\left(\mathrm{H}_{2} \mathrm{O}\right)\right]$}

\section{Meriem Benslimane, Yasmine Kheira Redjel, Hocine Merazig and Jean-Claude Daran}

\section{Computing details}

Data collection: COLLECT (Nonius, 1998); cell refinement: SCALEPACK (Otwinowski \& Minor, 1997); data reduction: DENZO and SCALEPACK (Otwinowski \& Minor, 1997); program(s) used to solve structure: SIR92 (Altomare et al., 1993); program(s) used to refine structure: SHELXL97 (Sheldrick, 2008); molecular graphics: ORTEP-3 for Windows (Farrugia, 2012) and DIAMOND (Brandenburg \& Berndt, 1999); software used to prepare material for publication:

Win $G X$ (Farrugia, 2012).

\section{Ammonium aquabis(sulfato)lanthanate(III)}

Crystal data

$\mathrm{NH}_{4}\left[\mathrm{La}\left(\mathrm{SO}_{4}\right)_{2}\left(\mathrm{H}_{2} \mathrm{O}\right)\right]$

$M_{r}=367.07$

Monoclinic, $P 2{ }_{1} / c$

Hall symbol: -P 2ybc

$a=8.4919$ (16) $\AA$

$b=9.978(2) \AA$

$c=11.9268(19) \AA$

$\beta=128.511(10)^{\circ}$

$V=790.7(3) \AA^{3}$

$Z=4$

\section{Data collection}

Nonius KappaCCD

diffractometer

Radiation source: fine-focus sealed tube

Graphite monochromator

Detector resolution: 9 pixels $\mathrm{mm}^{-1}$

CCD scans

Absorption correction: for a sphere

(Dwiggins, 1975)

$T_{\min }=0.419, T_{\max }=0.431$

Refinement

Refinement on $F^{2}$

Least-squares matrix: full

$R\left[F^{2}>2 \sigma\left(F^{2}\right)\right]=0.027$

$w R\left(F^{2}\right)=0.081$

$S=1.26$

2414 reflections

124 parameters

3 restraints
$F(000)=680$

$D_{\mathrm{x}}=3.083 \mathrm{Mg} \mathrm{m}^{-3}$

Mo $K \alpha$ radiation, $\lambda=0.71073 \AA$

Cell parameters from 2542 reflections

$\theta=3-30.5^{\circ}$

$\mu=5.96 \mathrm{~mm}^{-1}$

$T=100 \mathrm{~K}$

Prism, colourless

$0.30 \times 0.20 \times 0.10 \times 0.10$ (radius) $\mathrm{mm}$

2414 measured reflections

2414 independent reflections

2362 reflections with $I>2 \sigma(I)$

$R_{\text {int }}=0.0000$

$\theta_{\text {max }}=30.5^{\circ}, \theta_{\text {min }}=3.0^{\circ}$

$h=-12 \rightarrow 0$

$k=-14 \rightarrow 0$

$l=-12 \rightarrow 17$

Primary atom site location: structure-invariant direct methods

Secondary atom site location: difference Fourier map

Hydrogen site location: inferred from neighbouring sites

$\mathrm{H}$ atoms treated by a mixture of independent and constrained refinement 
$w=1 /\left[\sigma^{2}\left(F_{\mathrm{o}}^{2}\right)+(0.0427 P)^{2}+2.7376 P\right]$

where $P=\left(F_{\mathrm{o}}^{2}+2 F_{\mathrm{c}}^{2}\right) / 3$

$(\Delta / \sigma)_{\max }=0.001$
$\Delta \rho_{\max }=1.81 \mathrm{e} \AA^{-3}$
$\Delta \rho_{\min }=-1.48 \mathrm{e} \AA^{-3}$

\section{Special details}

Geometry. All e.s.d.'s (except the e.s.d. in the dihedral angle between two l.s. planes) are estimated using the full covariance matrix. The cell e.s.d.'s are taken into account individually in the estimation of e.s.d.'s in distances, angles and torsion angles; correlations between e.s.d.'s in cell parameters are only used when they are defined by crystal symmetry. An approximate (isotropic) treatment of cell e.s.d.'s is used for estimating e.s.d.'s involving 1.s. planes.

Refinement. Refinement of $F^{2}$ against ALL reflections. The weighted $R$-factor $w R$ and goodness of fit $S$ are based on $F^{2}$, conventional $R$-factors $R$ are based on $F$, with $F$ set to zero for negative $F^{2}$. The threshold expression of $F^{2}>\sigma\left(F^{2}\right)$ is used only for calculating $R$-factors(gt) etc. and is not relevant to the choice of reflections for refinement. $R$-factors based on $F^{2}$ are statistically about twice as large as those based on $F$, and $R$ - factors based on ALL data will be even larger.

Fractional atomic coordinates and isotropic or equivalent isotropic displacement parameters $\left(\AA^{2}\right)$

\begin{tabular}{lllll}
\hline & $x$ & $y$ & $z$ & $U_{\text {iso }} * / U_{\text {eq }}$ \\
\hline La1 & $0.71683(3)$ & $0.839390(18)$ & $0.248314(19)$ & $0.01052(8)$ \\
S1 & $0.74128(12)$ & $1.09162(8)$ & $0.42791(8)$ & $0.01163(15)$ \\
S2 & $0.70608(12)$ & $0.91270(8)$ & $-0.02026(8)$ & $0.01129(15)$ \\
O1 & $0.6085(4)$ & $1.0290(3)$ & $-0.1156(3)$ & $0.0179(5)$ \\
O2 & $0.8105(5)$ & $0.8337(3)$ & $-0.0602(3)$ & $0.0189(5)$ \\
O3 & $0.8535(4)$ & $0.9585(3)$ & $0.1310(3)$ & $0.0156(5)$ \\
O8 & $0.9057(4)$ & $1.1402(3)$ & $0.5727(3)$ & $0.0182(5)$ \\
O4 & $0.5597(4)$ & $0.8301(3)$ & $-0.0221(3)$ & $0.0188(5)$ \\
O7 & $0.5667(4)$ & $1.1797(3)$ & $0.3641(3)$ & $0.0229(6)$ \\
O6 & $0.6873(4)$ & $0.9516(3)$ & $0.4347(3)$ & $0.0185(5)$ \\
O5 & $0.8062(4)$ & $1.0870(3)$ & $0.3387(3)$ & $0.0192(5)$ \\
O1W & $0.8711(5)$ & $0.6537(3)$ & $0.2059(3)$ & $0.0241(6)$ \\
H11 & $0.982(5)$ & $0.615(6)$ & $0.266(4)$ & $0.036^{*}$ \\
H21 & $0.820(8)$ & $0.632(6)$ & $0.121(2)$ & $0.036^{*}$ \\
N1 & $0.2567(6)$ & $0.6458(4)$ & $-0.2302(4)$ & $0.0244(7)$ \\
& & & & \\
\hline
\end{tabular}

Atomic displacement parameters $\left(\AA^{2}\right)$

\begin{tabular}{lllllll}
\hline & $U^{11}$ & $U^{22}$ & $U^{33}$ & $U^{12}$ & $U^{13}$ & $U^{23}$ \\
\hline La1 & $0.01098(11)$ & $0.00981(11)$ & $0.01113(11)$ & $-0.00037(5)$ & $0.00706(9)$ & $-0.00028(5)$ \\
S1 & $0.0106(3)$ & $0.0110(3)$ & $0.0114(3)$ & $0.0009(3)$ & $0.0059(3)$ & $-0.0005(3)$ \\
S2 & $0.0118(3)$ & $0.0111(3)$ & $0.0101(3)$ & $0.0008(3)$ & $0.0064(3)$ & $0.0000(2)$ \\
O1 & $0.0212(12)$ & $0.0144(11)$ & $0.0173(11)$ & $0.0055(10)$ & $0.0116(10)$ & $0.0056(9)$ \\
O2 & $0.0214(13)$ & $0.0207(14)$ & $0.0180(13)$ & $0.0035(10)$ & $0.0140(12)$ & $-0.0013(9)$ \\
O3 & $0.0158(11)$ & $0.0162(11)$ & $0.0111(10)$ & $-0.0037(9)$ & $0.0066(9)$ & $-0.0028(9)$ \\
O8 & $0.0141(12)$ & $0.0206(12)$ & $0.0141(12)$ & $-0.0009(10)$ & $0.0059(10)$ & $-0.0041(10)$ \\
O4 & $0.0181(13)$ & $0.0212(13)$ & $0.0174(12)$ & $-0.0061(9)$ & $0.0111(11)$ & $-0.0018(9)$ \\
O7 & $0.0139(12)$ & $0.0207(13)$ & $0.0217(13)$ & $0.0068(10)$ & $0.0049(11)$ & $-0.0025(10)$ \\
O6 & $0.0240(13)$ & $0.0145(11)$ & $0.0174(12)$ & $-0.0029(10)$ & $0.0130(11)$ & $-0.0002(9)$ \\
O5 & $0.0246(13)$ & $0.0181(12)$ & $0.0211(12)$ & $-0.0020(10)$ & $0.0174(11)$ & $-0.0016(10)$ \\
O1W & $0.0289(16)$ & $0.0220(14)$ & $0.0177(13)$ & $0.0126(11)$ & $0.0127(12)$ & $0.0010(10)$ \\
N1 & $0.0248(16)$ & $0.0252(18)$ & $0.0296(18)$ & $-0.0037(13)$ & $0.0201(15)$ & $-0.0006(13)$ \\
& & & & & & \\
\hline
\end{tabular}


Geometric parameters $(\AA, \stackrel{\circ}{)})$

\begin{tabular}{|c|c|c|c|}
\hline $\mathrm{La} 1-\mathrm{O}^{\mathrm{i}}$ & $2.472(3)$ & $\mathrm{S} 1-\mathrm{O} 8$ & $1.471(3)$ \\
\hline $\mathrm{La} 1-\mathrm{O} 1 \mathrm{~W}$ & $2.496(3)$ & $\mathrm{S} 1-\mathrm{O} 5$ & $1.472(3)$ \\
\hline $\mathrm{La} 1-\mathrm{O}^{\mathrm{ii}}$ & $2.521(3)$ & $\mathrm{S} 1-\mathrm{O} 6$ & $1.488(3)$ \\
\hline $\mathrm{La} 1-\mathrm{O} 1^{\mathrm{iii}}$ & $2.533(3)$ & $\mathrm{S} 2-\mathrm{O} 1$ & $1.468(3)$ \\
\hline $\mathrm{La} 1-\mathrm{O} 2^{\mathrm{iv}}$ & $2.563(3)$ & $\mathrm{S} 2-\mathrm{O} 2$ & $1.470(3)$ \\
\hline $\mathrm{La} 1-\mathrm{O} 3$ & $2.596(3)$ & $\mathrm{S} 2-\mathrm{O} 4$ & $1.480(3)$ \\
\hline $\mathrm{La} 1-\mathrm{O} 5$ & $2.612(3)$ & $\mathrm{S} 2-\mathrm{O} 3$ & $1.490(3)$ \\
\hline $\mathrm{La} 1-\mathrm{O} 4$ & $2.614(3)$ & $\mathrm{O} 1 \mathrm{~W}-\mathrm{H} 11$ & $0.845(10)$ \\
\hline La1-O6 & $2.637(3)$ & $\mathrm{O} 1 \mathrm{~W}-\mathrm{H} 21$ & $0.844(10)$ \\
\hline $\mathrm{S} 1-\mathrm{O} 7$ & $1.465(3)$ & & \\
\hline $\mathrm{O} 7{ }^{\mathrm{i}}-\mathrm{La} 1-\mathrm{O} 1 \mathrm{~W}$ & $82.44(12)$ & $\mathrm{O} 7^{\mathrm{i}}-\mathrm{La} 1-\mathrm{O} 6$ & $99.16(10)$ \\
\hline 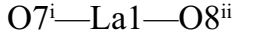 & $143.78(10)$ & $\mathrm{O} 1 \mathrm{~W}-\mathrm{La} 1-\mathrm{O} 6$ & $145.43(9)$ \\
\hline $\mathrm{O} 1 \mathrm{~W}-\mathrm{La} 1-\mathrm{O} 8^{\mathrm{ii}}$ & $71.36(10)$ & $\mathrm{O} 8^{\mathrm{ii}}-\mathrm{La} 1-\mathrm{O} 6$ & $89.43(9)$ \\
\hline 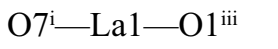 & $71.36(10)$ & $\mathrm{O} 1{ }^{\mathrm{iii}}-\mathrm{La} 1-\mathrm{O} 6$ & $70.57(9)$ \\
\hline $\mathrm{O} 1 \mathrm{~W}-\mathrm{La} 1-\mathrm{O} 1^{\mathrm{iii}}$ & $139.83(10)$ & $\mathrm{O} 2^{\mathrm{iv}}-\mathrm{La} 1-\mathrm{O} 6$ & $71.00(9)$ \\
\hline 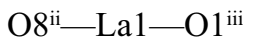 & $143.55(9)$ & $\mathrm{O} 3-\mathrm{La} 1-\mathrm{O} 6$ & $124.69(8)$ \\
\hline 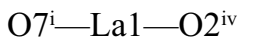 & $72.90(10)$ & $\mathrm{O} 5-\mathrm{La} 1-\mathrm{O} 6$ & $53.55(8)$ \\
\hline $\mathrm{O} 1 \mathrm{~W}-\mathrm{La} 1-\mathrm{O} 2^{\mathrm{iv}}$ & $76.67(10)$ & $\mathrm{O} 4-\mathrm{La} 1-\mathrm{O} 6$ & $144.28(9)$ \\
\hline $\mathrm{O} 8^{\mathrm{ii}}-\mathrm{La} 1-\mathrm{O} 2^{\mathrm{iv}}$ & $76.96(10)$ & $\mathrm{O} 7-\mathrm{S} 1-\mathrm{O} 8$ & $109.04(17)$ \\
\hline 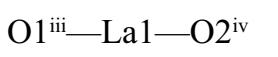 & $121.24(9)$ & $\mathrm{O} 7-\mathrm{S} 1-\mathrm{O} 5$ & $110.89(19)$ \\
\hline $\mathrm{O} 7$ - $-\mathrm{La} 1-\mathrm{O} 3$ & $127.89(10)$ & $\mathrm{O} 8-\mathrm{S} 1-\mathrm{O} 5$ & $110.49(17)$ \\
\hline $\mathrm{O} 1 \mathrm{~W}-\mathrm{La} 1-\mathrm{O} 3$ & $76.32(10)$ & $\mathrm{O} 7-\mathrm{S} 1-\mathrm{O} 6$ & $110.19(18)$ \\
\hline $\mathrm{O} 8^{\mathrm{ii}}-\mathrm{La} 1-\mathrm{O} 3$ & $70.11(9)$ & $\mathrm{O} 8-\mathrm{S} 1-\mathrm{O} 6$ & $110.17(16)$ \\
\hline $\mathrm{O} 1{ }^{\mathrm{iii}-\mathrm{La} 1-\mathrm{O} 3}$ & $96.07(9)$ & $\mathrm{O} 5-\mathrm{S} 1-\mathrm{O} 6$ & $106.04(16)$ \\
\hline $\mathrm{O} 2^{\mathrm{iv}}-\mathrm{La} 1-\mathrm{O} 3$ & $142.55(9)$ & $\mathrm{O} 7-\mathrm{S} 1-\mathrm{La} 1$ & $119.80(13)$ \\
\hline O7-La1-O5 & $140.16(10)$ & $\mathrm{O} 8-\mathrm{S} 1-\mathrm{La} 1$ & $131.15(12)$ \\
\hline $\mathrm{O} 1 \mathrm{~W}-\mathrm{La} 1-\mathrm{O} 5$ & $137.02(11)$ & $\mathrm{O} 5-\mathrm{S} 1-\mathrm{La} 1$ & $52.71(11)$ \\
\hline $\mathrm{O} 8^{\mathrm{ii}-\mathrm{La} 1-\mathrm{O} 5}$ & $71.62(9)$ & O6-S1-La1 & $53.78(11)$ \\
\hline $\mathrm{O}{ }^{\mathrm{iii}}-\mathrm{La} 1-\mathrm{O} 5$ & $72.00(9)$ & $\mathrm{O} 1-\mathrm{S} 2-\mathrm{O} 2$ & $109.67(16)$ \\
\hline $\mathrm{O} 2^{\mathrm{iv}}-\mathrm{La} 1-\mathrm{O} 5$ & $114.84(9)$ & $\mathrm{O} 1-\mathrm{S} 2-\mathrm{O} 4$ & $111.40(17)$ \\
\hline $\mathrm{O} 3-\mathrm{La} 1-\mathrm{O} 5$ & $71.17(8)$ & $\mathrm{O} 2-\mathrm{S} 2-\mathrm{O} 4$ & $111.52(17)$ \\
\hline 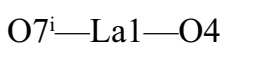 & $74.43(10)$ & $\mathrm{O} 1-\mathrm{S} 2-\mathrm{O} 3$ & $109.85(16)$ \\
\hline $\mathrm{O} 1 \mathrm{~W}-\mathrm{La} 1-\mathrm{O} 4$ & $69.69(10)$ & $\mathrm{O} 2-\mathrm{S} 2-\mathrm{O} 3$ & $109.59(17)$ \\
\hline 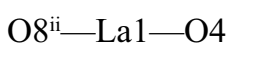 & $116.81(9)$ & $\mathrm{O} 4-\mathrm{S} 2-\mathrm{O} 3$ & $104.70(16)$ \\
\hline $\mathrm{O} 1 \mathrm{ii}-\mathrm{La} 1-\mathrm{O} 4$ & $74.17(9)$ & $\mathrm{La} 1-\mathrm{O} 1 \mathrm{~W}-\mathrm{H} 11$ & $128(4)$ \\
\hline $\mathrm{O} 2^{\mathrm{iv}}-\mathrm{La} 1-\mathrm{O} 4$ & $135.44(9)$ & $\mathrm{La} 1-\mathrm{O} 1 \mathrm{~W}-\mathrm{H} 21$ & $119(4)$ \\
\hline $\mathrm{O} 3-\mathrm{La} 1-\mathrm{O} 4$ & $53.65(8)$ & $\mathrm{H} 11-\mathrm{O} 1 \mathrm{~W}-\mathrm{H} 21$ & $112(3)$ \\
\hline $\mathrm{O} 5-\mathrm{La} 1-\mathrm{O} 4$ & $109.69(9)$ & & \\
\hline
\end{tabular}

Symmetry codes: (i) $-x+1, y-1 / 2,-z+1 / 2$; (ii) $-x+2,-y+2,-z+1$; (iii) $-x+1,-y+2,-z$; (iv) $x,-y+3 / 2, z+1 / 2$.

Hydrogen-bond geometry $\left(A,{ }^{\circ}\right)$

\begin{tabular}{lllll}
\hline$D-\mathrm{H} \cdots A$ & $D-\mathrm{H}$ & $\mathrm{H} \cdots A$ & $D \cdots A$ & $D-\mathrm{H} \cdots A$ \\
\hline $\mathrm{O} 1 W-\mathrm{H} 11 \cdots \mathrm{O}^{\mathrm{v}}$ & $0.84(5)$ & $1.94(5)$ & $2.717(5)$ & $153(5)$ \\
$\mathrm{O} 1 W-\mathrm{H} 21 \cdots$ O6 $^{\mathrm{vi}}$ & $0.85(3)$ & $1.95(3)$ & $2.778(4)$ & $168(5)$
\end{tabular}




\section{supporting information}

$\begin{array}{ll}\mathrm{N} 1 \cdots \mathrm{O} 1^{\text {vii }} & 2.942(5) \\ \mathrm{N} 1 \cdots 6^{\text {vi }} & 3.036(5) \\ \mathrm{N} 1 \cdots 3^{\text {viii }} & 2.914(5) \\ \mathrm{N} 1 \cdots 8^{\mathrm{i}} & 2.943(5) \\ \mathrm{N} 1 \cdots \mathrm{O} 5^{\text {iii }} & 2.865(5) \\ \mathrm{N} 1 \cdots \mathrm{O} 4 & 2.866(5)\end{array}$

Symmetry codes: (i) $-x+1, y-1 / 2,-z+1 / 2$; (iii) $-x+1,-y+2,-z$; (v) $-x+2, y-1 / 2,-z+1 / 2$; (vi) $x,-y+3 / 2, z-1 / 2$; (vii) $-x+1, y-1 / 2,-z-1 / 2$; (viii) $x-1$, $-y+3 / 2, z-1 / 2$. 